\title{
Hematite-based Photo-oxidation of Water Using Transparent Distributed Current Collectors
}

\author{
Shannon C. Riha, ${ }^{\dagger \neq, \|}$ Michael J. DeVries Vermeer, ${ }^{\dagger, \S, \|}$ Michael J. Pellin, ${ }^{\dagger \dagger, \S}$ Joseph T. Hupp, ${ }^{* \dagger, t, \S}$ \\ and Alex B. F. Martinson*,t, \\ ${ }^{\dagger}$ Argonne-Northwestern Solar Energy Research (ANSER) Center and ${ }^{\S}$ Department of Chemistry, Northwestern University, \\ Evanston, Illinois 60208, United States \\ ${ }^{\ddagger}$ Materials Science Division, Argonne National Laboratory, Argonne, Illinois 60439, United States
}

\section{Supporting Information}

\begin{abstract}
High specific surface area transparent and conducting frameworks, fabricated by atomic layer deposition (ALD), were used as scaffolds for fabrication of equally high area, ALD-formed hematite structures for photo-oxidation of water to dioxygen. The frameworks offer high transparency to visible light and robust conductivity under harsh annealing and oxidizing conditions. Furthermore, they also make possible the spatially distributed collection of photocurrent from ultrathin coatings of hematite layers, enabling the formation of photoanodes featuring both large optical extinction and a hematite layer thickness nearly commensurate with the hole-collection distance. The distributed-current-collection approach increases the efficiency of water oxidation with hematite (by about a factor of 3 compared with an optimized flat electrode), is highly adaptable to future advances in thin film technology, and is further

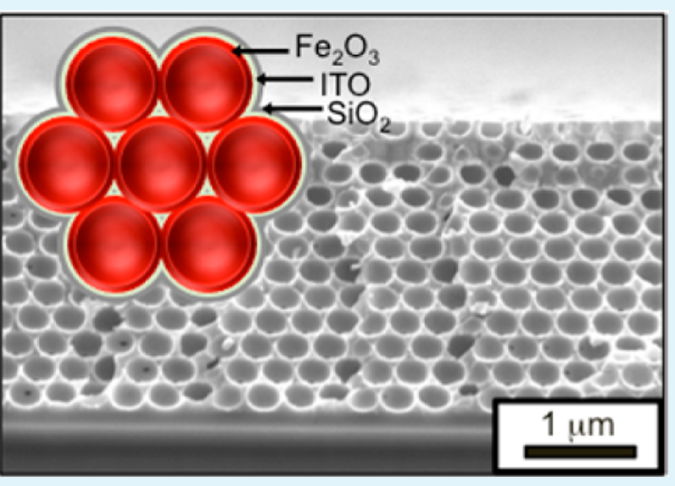
applicable to a multitude of nanostructures and optoelectronic applications that require ultrathin films without sacrificing optical thickness.
\end{abstract}

KEYWORDS: atomic layer deposition, $\mathrm{Fe}_{2} \mathrm{O}_{3}$, inverse opal, iron oxide, water splitting, distributed current collector

\section{INTRODUCTION}

The sun irradiates the earth's surface with more than enough power to sustainably meet the rapidly growing energy demand, but large scale solar energy utilization is constrained by two key challenges: supplying energy while the sun is not shining (i.e., energy storage) and efficiently converting solar radiation using more affordable materials. ${ }^{1}$ One promising approach to surmounting both is the direct photoelectrochemical generation of fuels from sunlight (e.g., solar water splitting) using earth-abundant materials. ${ }^{1-6}$ One such earth-abundant material, hematite $\left(\alpha-\mathrm{Fe}_{2} \mathrm{O}_{3}\right)$, has received renewed interest as a viable photoanode for water oxidation, half of the overall watersplitting reaction. ${ }^{5,7-18}$ Hematite has a band gap of $2.1 \mathrm{eV}$, which allows for light absorption out to approximately $600 \mathrm{~nm}$, and a valence band edge suitably positioned for water oxidation. The nontoxic, $n$-type semiconductor is a form of iron oxide, with Fe being in the fully oxidized (III) state, that is remarkably stable under the often alkaline and oxidizing conditions used when splitting water. Despite its promise, the careful study of hematite photoanodes has revealed two significant limitations that have impeded widespread application. First, polycrystalline hematite surfaces show only modest activity for the oxygen evolution reaction (OER) at the semiconductor-liquid junction $^{14,17}$ and second, the observed hole-collection distance is typically less than $20 \mathrm{~nm} \cdot{ }^{10,11,19-22}$
The incongruity between hole collection distances and optical absorption length for hematite is illustrated in Figure 1. Photogenerated holes are swept to the semiconductor-liquid junction under the influence of a space-charge layer, which has been reported to have a width similar to the distance of hole collection. ${ }^{10,22}$ Holes produced outside this drift-assisted region are generally lost to rapid recombination, making films thicker than the depletion width no more productive than thinner films and worse, acting as optical filters to the active region under glass-side illumination. Therefore, while a relatively thick film (hundreds of nanometers) is needed to achieve light harvesting efficiencies (LHE) approaching unity, in practice thick films show external quantum efficiencies equal to or less than thin film $(\sim 20 \mathrm{~nm})$ planar photoanodes. ${ }^{9,10}$ Nanostructuring of photoelectrodes could provide a means of decoupling the small feature sizes required for effective collection of photogenerated holes from the relatively large optical thickness needed for efficient light-harvesting.

Two approaches for nanostructuring hematite are considered: the first entails changing the morphology of hematite monolith, while the second entails scaffolding hematite thin

\footnotetext{
Received: October 16, 2012

Accepted: December 12, 2012

Published: January 3, 2013
} 


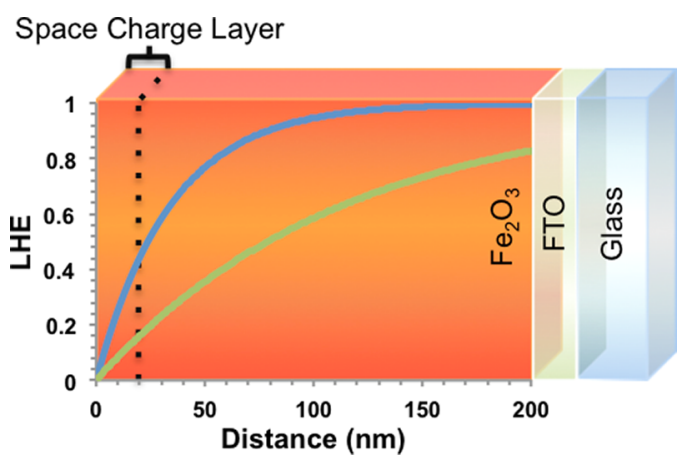

Figure 1. (a) Absorptance profile of $400 \mathrm{~nm}$ (blue) and $525 \mathrm{~nm}$ (green) light in a dense hematite thin film based on $1-10^{(-\alpha \mathrm{d})}$. More than $200 \mathrm{~nm}$ of hematite is required to absorb most green photons. Less than $50 \%$ of any visible light is absorbed within the space-charge layer, where an electric field assists the transport of photogenerated holes to the semiconductor-liquid junction.

films on conductive nanostructured supports. Efforts to nanostructure hematite through morphological controlincluding the formation of nanoparticles, ${ }^{23}$ nanorods, ${ }^{24}$ nanowire arrays, ${ }^{25-27}$ and cauliflower-like structures made with atmospheric pressure chemical vapor deposition ${ }^{28}$ - have resulted in improved device performance. Each of these structuring strategies improves light collection at longer wavelengths by making an optically thicker, porous hematite layer while minimizing the distance photogenerated holes must travel to the solid-liquid junction. Nanostructuring the semiconductor circumvents hematite's conflicting demands for relatively long optical thickness and short hole-collection distance. However, in the aforementioned nanostructures the potential gradient associated with the space-charge layer sweeps electrons not into the electrode directly but first into the interior of the hematite nanostructure. If the space-charge layer does not extend fully into the interior it may contribute to (parasitic) absorption without contributing to photocurrent. An example of this point was clearly demonstrated by Klahr, et al. with hematite films grown by atomic layer deposition (ALD). They found that films thicker than 20 showed higher absorbance values but did not correlate with increased photocurrent. ${ }^{10}$ Compared to electrons traversing ultrathin films, these charges must also travel a longer and more tortuous path to the collecting electrode, thereby increasing their interaction time with nearby holes.

A more experimentally challenging but conceptually simpler design can be envisioned whereby a transparent current collector, which covers an inert and transparent framework, is directly adjacent to all points of electron generation, as is the case for flat thin films. This approach eliminates competitive absorption (from unproductive $\mathrm{Fe}_{2} \mathrm{O}_{3}$ ), minimizes the electron collection distance (and therefore interaction time), and ameliorates concerns about potential-draining series resistance. However, the requirements for this scaffold-essentially, a spatially distributed current collector-are not trivial. ${ }^{29-32}$ Clearly, the framework needs to be conductive to minimize the resistance to electron flow. Second, this scaffold must be sufficiently porous to allow reactants and products access to the deepest pores of the photoanode. Next, the material must be compatible with the deposition and postprocessing of subsequent layers (hematite) as well as resistant to a water oxidation environment, assuming a few pinholes may always be present in the ultrathin films. Finally, and perhaps most challenging, is the requirement for the high optical transparency over the range of near-UV and visible-light wavelengths absorbed by hematite $(350-600 \mathrm{~nm})$. Frameworks that meet some, but not all, of these requirements have been fabricated from anodized aluminum oxide templates, ${ }^{33} \mathrm{TiSi}_{2}$ nanonets, ${ }^{13}$ and $\mathrm{Al}: \mathrm{ZnO}$ nanorods. ${ }^{11,32}$ Subsequent coating of the conductive scaffolds with ALD thin films of hematite resulted in photoanodes with improved photocurrent density but less than optimal electrical, optical, and/or materials properties. During the preparation of this manuscript, a report describing an ALD-grown $\mathrm{Nb}: \mathrm{SnO}_{2}$ thin film over $\mathrm{TiO}_{2}$ nanoparticles was published by Stefik, et al. ${ }^{32}$ This distributed electrode was subsequently coated with hematite, but not by ALD, resulting in a nonuniform $\mathrm{Fe}_{2} \mathrm{O}_{3}$ coating on a framework of modest porosity. Nevertheless, the large improvement in water splitting performance they observe further reinforces the promise of this approach.

Herein, we report a materials-general approach to the deployment of transparent and conductive scaffolds constructed by conformally coating silica-based inverse opal frameworks with indium tin oxide (ITO) via ALD. Subsequent deposition of $\mathrm{Fe}_{2} \mathrm{O}_{3}$, also by $\mathrm{ALD}$, results in an efficient photoanode for water oxidation with the flexibility to accommodate future improvements in both the transparentconducting and absorbing-semiconducting components. The inverse-opal design offers a high degree of tunability, with the ability to easily vary pore size and total thickness. Additionally, the ITO and hematite layer thicknesses (and thus conductance and light harvesting efficiency, respectively) can be varied independently and with atomic layer precision contrary to hematite films grown by other methods. In principle, these layers could be similarly grown on other transparent, porous frameworks. When employed as water oxidation photoanodes we observe a more favorable photocurrent-onset potential, as well as enhancements of light harvesting efficiency-correlated directly to increases in external quantum efficiency (EQE)relative to equivalent flat photoanodes.

\section{EXPERIMENTAL SECTION}

Conductive fluorine-doped tin oxide (FTO) coated glass $\left(15 \Omega \mathrm{cm}^{-2}\right.$ ) was purchased from Hartford Glass Co. Substrates were cleaned by sonicating in deionized (DI) water and detergent for $20 \mathrm{~min}$, rinsing with DI water, sonicating in acetone, rinsing with acetone, sonicating in isopropanol, and finally blowing dry with $\mathrm{N}_{2}$. Porous inverse opal thick films were fabricated according to modified literature procedure that will be reported in detail elsewhere. ${ }^{34,35}$ The procedure uses a $2.6 \%$ stock solution of $0.350 \mu \mathrm{m}$ polystyrene particles.

ITO and $\mathrm{Fe}_{2} \mathrm{O}_{3}$ deposition was performed in a Savannah 200 ALD (Cambridge Nanotech Inc.). Ferrocene $\left(\mathrm{Fe}(\mathrm{Cp})_{2}, 98 \%\right.$, Aldrich), cyclopentadienylindium(I) (InCp, 99.99\%, Strem), tetrakis(dimethylamido)tin(IV) (TDMASn, 99.9\%, Aldrich) and ozone $\left(\mathrm{O}_{3}\right)$ were used as precursors. Ozone was generated by a DelOzone ozone generator ( $5 \mathrm{wt} \%$ in $500 \mathrm{sccm}$ ultrahigh purity oxygen). For the ITO deposition, InCp was heated to $70{ }^{\circ} \mathrm{C}$ and the TDMASn to 55 ${ }^{\circ} \mathrm{C}$, whereas substrates were heated to $210^{\circ} \mathrm{C}$. Sn-doping was achieved using a cycle ratio of 9:1 for $\mathrm{In}_{2} \mathrm{O}_{3}$ and $\mathrm{SnO}_{2}$, respectively. For the deposition of $\operatorname{In}_{2} \mathrm{O}_{3}$, the ALD pulse sequence was $30 \mathrm{~s}$ InCp static exposure (no pumping) followed by a $35 \mathrm{~s}$ purge, $65 \mathrm{~s} \mathrm{O}_{3}$ pulse, and finally a $25 \mathrm{~s}$ purge. The $\mathrm{SnO}_{2}$ deposition followed a similar recipe: 15 $\mathrm{s}$ TDMASn exposure, $30 \mathrm{~s}$ purge, $65 \mathrm{~s}_{3}$ pulse, and a $25 \mathrm{~s}$ purge. No postannealing of the ITO-coated scaffold was necessary. The ferrocene precursor was heated to $95{ }^{\circ} \mathrm{C}$ and the substrates cooled slightly to $200{ }^{\circ} \mathrm{C}$ for the deposition of $\mathrm{Fe}_{2} \mathrm{O}_{3}$. The pulse sequence (a modification of a previously published procedure $)^{36-39}$ consisted of the following: two $30 \mathrm{~s} \mathrm{Fe}(\mathrm{Cp})_{2}$ exposures separated by a $5 \mathrm{~s}$ purge 
and followed by a $35 \mathrm{~s}$ purge, then two $60 \mathrm{~s}$ ozone exposures separated by a $5 \mathrm{~s}$ purge and followed by a $15 \mathrm{~s}$ purge. The nitrogen carrier gas flow rate was $15 \mathrm{sccm}$ during purge times and $5 \mathrm{sccm}$ during quasistatic exposures. A portion of the hematite-coated substrate that was not coated with opal was masked by Kapton tape during deposition to reduce the contact resistance of the potentiostat leads. All photoanodes were subsequently annealed at $500{ }^{\circ} \mathrm{C}$ for $30 \mathrm{~min}$ with $10{ }^{\circ} \mathrm{C} /$ min ramp rates. ITO and hematite film thicknesses were estimated from ellipsometry measurements of coatings formed on witness silicon substrates using a J. A. Woolam Co. M2000 variable angle spectroscopic ellipsometer (VASE). Scanning electron microscope (SEM) images and energy-dispersive X-ray spectra (EDS) were acquired on a Hitachi S4800-II or Hitachi S4700 scanning electron microscope.

Photoelectrochemical measurements were performed with a $\mu$ Autolab Type III potentiostat with NOVA software, in $1 \mathrm{M} \mathrm{NaOH}$ deionized water ( $\mathrm{pH}$ 13.6) with a platinum wire counter-electrode and $\mathrm{Ag} / \mathrm{AgCl} /$ sat. $\mathrm{KCl}$ reference electrode. The reported potential was converted to be in reference to the reversible hydrogen electrode (RHE) - as it is independent of the electrolyte $\mathrm{pH}$ - using the relationship:

$$
E_{\mathrm{RHE}}=E_{\mathrm{Ag} / \mathrm{AgCl}}+0.059 \mathrm{pH}+E_{\mathrm{Ag} / \mathrm{AgCl}}^{0}
$$

Here, $E_{\mathrm{Ag} / \mathrm{AgCl}}$ is the measured potential using the $\mathrm{Ag} / \mathrm{AgCl}$ reference electrode, and $E_{\mathrm{Ag} / \mathrm{AgCl}}^{0}$ is the standard potential of the $\mathrm{Ag} / \mathrm{AgCl}$ reference electrode vs NHE $(0.197 \mathrm{~V})$. The light source was a Newport $300 \mathrm{~W}$ arc lamp equipped with an AM 1.5 and ultraviolet (UV) filter interfaced with a Newport monochromator with switchable grating or mirror (for white light illumination). The output power was calibrated with a Newport silicon photodiode to roughly simulate AM 1.5 conditions $\left(100 \mathrm{~mW} \mathrm{~cm}^{-2}\right)$. Substrates masked with Surlyn, which defines an active area of $0.38 \mathrm{~cm}^{2}$, were immersed in solution and illuminated through a quartz window through the hematite side of the photoanode.

Reflectance-corrected absorbance spectra were recorded with a Cary 5000 UV-vis-NIR fitted with an integrating sphere accessory (DRA2500). For both the attenuance and reflectance measurements, dry samples were illuminated through the glass side of the substrate using an optical mask to prevent light leaking. The FTO substrate transmittance and reflectance were subtracted from the spectra to yield the transmittance and reflectance of only the $\mathrm{ITO} / \mathrm{Fe}_{2} \mathrm{O}_{3}$ layer. Using the relationship $\mathrm{Abs}=-\log (T+R)$, the absorbance of the ITO/ $\mathrm{Fe}_{2} \mathrm{O}_{3}$ active layer was then determined. Absorptance was derived using the equation $1-10^{-\mathrm{Abs}}$ or otherwise put, absorptance $=1-T-$ $R$.

A detailed analysis of the photon flow through flat and scaffolded hematite photoanodes was calculated for 400 and $525 \mathrm{~nm}$. We took measurements of attenuance and \% reflectance for each of the samples with the UV-vis-NIR spectrometer, before and after deposition of ITO and $\mathrm{Fe}_{2} \mathrm{O}_{3}$, in addition to EQE measurements. The \% reflected by the substrate was found simply from the \% reflectance measurements. Transmitted photons were calculated from (1 - reflectance attenuation from the substrate). Active layer absorbtance for flat samples was calculated by subtracting predeposition (base) absorbtance from postdeposition (base+active) absorbtance. The \% absorbed by FTO on flat substrates was calculated by multiplying the total absorbtance of the FTO substrate (measured predeposition) by the \% of incident light not reflected by the substrate or absorbed by $\mathrm{Fe}_{2} \mathrm{O}_{3}$. Finally, the \% collected was a known quantity from EQE measurements taken at $1.53 \mathrm{~V}$ vs RHE. These data were found by subtracting dark current from photocurrent at the wavelength of interest, then dividing the result by incident power from the lamp. In the figure, \% recombined was then calculated by subtracting \% collected from \% absorbed by $\mathrm{Fe}_{2} \mathrm{O}_{3}$.

\section{RESULTS AND DISCUSSION}

Given the strict requirements for an effectual scaffold, and the chemically aggressive environment to which it will be exposed, we sought to establish its properties alone under these experimental conditions. Previous studies of ALD-grown hematite thin films indicate that firing at $500{ }^{\circ} \mathrm{C}$ under flowing $\mathrm{O}_{2}$ optimizes photoanode performance. ${ }^{9-13,18,40}$ Our studies with various firing times suggested that $30 \mathrm{~min}$ was optimal for hematite thin films. However, commercial ITO films and many other transparent conducting oxides (TCOs) are known to lose conductivity under these conditions. ${ }^{11}$ High-temperature treatments may increase grain size in weakly crystalline or amorphous films, and therefore allow for higher charge mobility. However, the conductivities of TCOs are highly sensitive to $\mathrm{O}_{2}$ partial pressure at elevated temperatures, which dramatically changes the concentration of O-vacancies that account for $n$-type doping. When thin films of pure $\operatorname{In}_{2} \mathrm{O}_{3}$ grown by ALD were subjected to the annealing conditions used throughout this work they became highly resistive due to a reduction in the carrier concentration that likely corresponds to a lower O-vacancy concentration. Sn-doping of $\operatorname{In}_{2} \mathrm{O}_{3}$ is also known to create $n$-type carriers, and thus diminishes (but does not eliminate) the sensitivity of film conductivity to thermal elimination of $\mathrm{O}$-vacancies. Nevertheless, retaining substantial conductivity is a near-universal challenge for TCOs at $500{ }^{\circ} \mathrm{C}$ in oxygen (perhaps only FTO being excluded) that is even more pronounced in $\mathrm{Al}: \mathrm{ZnO}$ films. ${ }^{41}$

To observe how the electrical conductance of the ITO scaffold changed throughout photoanode construction, we fabricated a special substrate (Figure 2). A $\sim 5 \mathrm{~mm}$ gap was

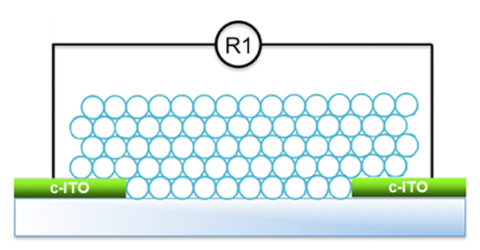

Figure 2. Highly idealized schematic of the special substrate for measuring resistance across the ALD-ITO-coated inverse opal framework. The schematic is not to scale. Actual inverse opal thickness is of order $5 \mu \mathrm{m}$, whereas the gap between ITO pads is on the order of $5 \mathrm{~mm}$.

chemically etched down the center of commercial ITO-coated (cITO) glass substrate, leaving only two electrically isolated strips of ITO. An inverse opal was then assembled across the full width of the substrate, followed by ALD growth of ITO (aITO) and $\mathrm{Fe}_{2} \mathrm{O}_{3}$. Resistances were measured at each stage of fabrication to monitor how the scaffold conductance changed. Assurance that the conductance was retained cannot be provided in the traditional device geometry (no cITO etching) because of the high contact resistance of the $\mathrm{Fe}_{2} \mathrm{O}_{3}$-coating and the risk of probe breakthrough onto the underlying TCO. Table 1 shows the resistance to lateral electrical conduction across the inverse opal framework (R1) at various stages of fabrication.

As expected, the uncoated silica inverse opal framework was highly insulating. After conformal deposition of $8 \mathrm{~nm}$ of aITO, the porous framework became conductive, exhibiting a lateral resistance of $150 \Omega$. We note that the relevant resistance (for electron collection to the typically underlying electrode, through the few micrometer thickness of the porous inverse opal scaffold) will be orders of magnitude lower according to the geometric relationship of resistance. Remarkably, following annealing under the same conditions used for annealing $\mathrm{Fe}_{2} \mathrm{O}_{3}$, conductivity across the array was unaltered. Deposition of 
Table 1. Electrical Resistance at Difference Stages of Device Fabrication for the Geometry Shown in Figure $2^{a}$

\begin{tabular}{ll}
\multicolumn{1}{c}{ sample } & \multicolumn{1}{c}{$\mathrm{R} 1$} \\
$\mathrm{cITO} / \mathrm{IO}$ & $\infty$ \\
$\mathrm{cITO} / \mathrm{IO} / \mathrm{aITO}$ & $150 \Omega$ \\
$\mathrm{cITO} / \mathrm{IO} / \mathrm{aITO}+500{ }^{\circ} \mathrm{C} \mathrm{O}_{2}$ & $150 \Omega$ \\
$\mathrm{cITO} / \mathrm{IO} / \mathrm{aITO} / \mathrm{Fe}_{2} \mathrm{O}_{3}$ & $195 \Omega$ \\
$\mathrm{cITO} / \mathrm{IO} / \mathrm{aITO} / \mathrm{Fe}_{2} \mathrm{O}_{3}+500{ }^{\circ} \mathrm{C} \mathrm{O}_{2}$ & $218 \Omega$
\end{tabular}

${ }^{a}$ cITO $=$ commercial ITO substrate, aITO $=$ ALD-grown ITO on the inverse opals (IO).

$\mathrm{Fe}_{2} \mathrm{O}_{3}$ onto an cITO/IO/aITO substrate produces a relatively small increase in resistance. After annealing in $\mathrm{O}_{2}$ the final photoanode showed a sheet resistance on the order of $200 \Omega$. Therefore, we conclude that none of the processing conditions to which real photoelectrodes will be subjected, seriously erodes the conductivity of these high surface area scaffolds. The resistance to vertical electrical conduction, which is most relevant to device operation (electron collection), is difficult to reliably measure because of the risk of probe breakthrough and "sealing" of all exposed surfaces by $\mathrm{Fe}_{2} \mathrm{O}_{3}$. Instead, to the extent that conduction is symmetric in the largely symmetric inverseopal, we expect the resistance to be much less than $1 \Omega$ through the scaffold, ${ }^{42}$ even after postdeposition processing. This resistance is negligible compared to the sheet resistance of the substrate (nominally $15 \mathrm{ohms}$ ).

After verification of the conductive scaffold properties we optimized the hematite thickness in planar FTO devices. Very thin, flat hematite films $(<10 \mathrm{~nm})$ yielded negligible photocurrents even after high temperature firing in air. The absorption spectra of these films tended to show comparatively poorly defined peaks, suggesting a lack of crystallinity, even after firing. This highlights the need for a precise and conformal iron oxide film throughout the framework in order to avoid formation of "dead zones", i.e. regions where the film might absorb light but be unable to generate photocurrent, thus lowering the overall efficiency. Likewise, deposition that results in areas that are overly thick could result in closed pores, making portions of the semiconductor film inaccessible to water and therefore inactive. Thus, the structural precision and conformality of ALD-grown films is uniquely suited to this device design and application. Cross-sectional scanning electron microscopy (SEM) was undertaken to probe the precision of deposition throughout the challenging structure.

The SEM images (Figure 3 and Figure S2 in the Supporting Information) show conformal nanolaminates of uniform thickness deposited throughout the eight-layer inverse opal structure. A film of approximately $20 \mathrm{~nm}$ is clearly visible around the inside of each cavity, with the thinner ITO layer distinguishable beneath. Although 15-nm-thick films were found to be optimal for photoelectrochemical water oxidation, intentionally thicker hematite films were grown over the ITO in this study to allow better imaging and test the limits of conformal growth. Figure $3 \mathrm{c}$ suggests that adequate contact is made between the aITO/hematite film and the underlying FTO substrate.

The apertures between cavities, visible in Figure $3 \mathrm{a}, \mathrm{b}$ are approximately $30-40 \mathrm{~nm}$ in diameter at their widest and appear to be unbarred by the film deposition. If pore closing by the ALD growth were to occur, it would render those cavities inactive due to the inability of the electrolyte to penetrate the pores. Additionally, deposition would be inhibited deep within
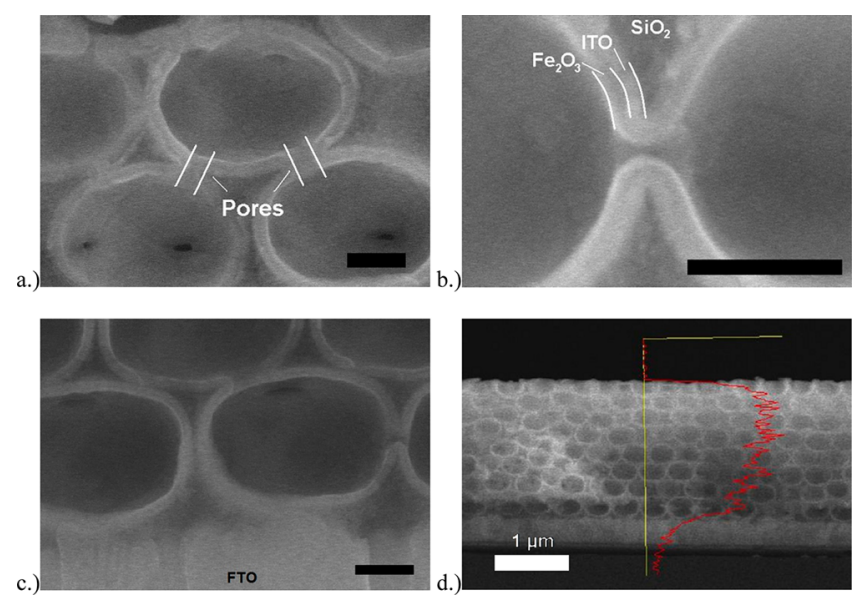

Figure 3. $(\mathrm{a}-\mathrm{c})$ SEM cross-section of inverse opal scaffolds coated with ITO and $20 \mathrm{~nm} \mathrm{Fe} \mathrm{O}_{3}$. The black bar represents $100 \mathrm{~nm}$ in each image. (d) Overlayed EDS linescan showing iron deposited throughout the film thickness.

the framework, yielding thicker ALD films near the surface and little or no deposition near the substrate. Further evidence that $\mathrm{Fe}_{2} \mathrm{O}_{3}$ deposition occurred even in the deepest pores of the scaffold is supported by an EDS linescan of Fe, collected over a cross-section of the scaffold, Figure 3d. Combined, the SEM and EDS analysis provide strong evidence for the final deposition of $\mathrm{Fe}_{2} \mathrm{O}_{3}$ by ferrocene and ozone, resulting in a uniform and conformal thin film.

Reflectance-corrected UV-vis spectra were collected for dry photoanodes at each stage of fabrication and for several control samples. A simple comparison of equivalent $\alpha-\mathrm{Fe}_{2} \mathrm{O}_{3}$ thicknesses on nominally flat FTO and inverse opal (without ITO) substrates is most illustrative, Figure 4. A three- to 6-fold

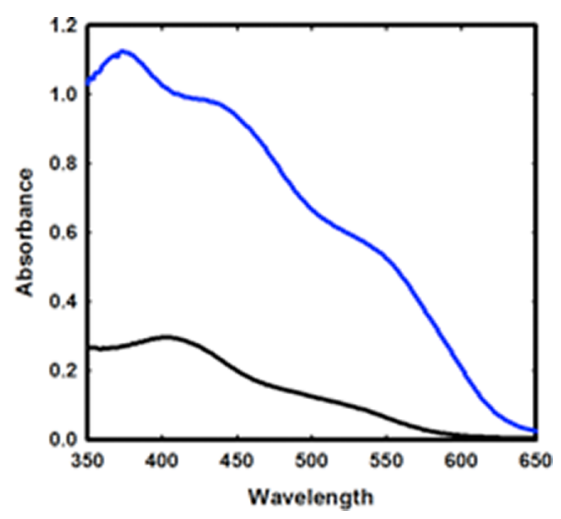

Figure 4. Absorbance of $15 \mathrm{~nm} \mathrm{Fe}_{2} \mathrm{O}_{3}$ on an inverse opal (blue) and on a flat FTO substrate (black) after firing at $500{ }^{\circ} \mathrm{C}$.

increase in absorbance is observed for the inverse opal sample over the wavelength range between 350 and $550 \mathrm{~nm}$ (Figures 4 and 6). A much more detailed optical analysis that includes the reflectivity of each photoanode as well as competitive absorption by the ITO-coated inverse opal is required to predict the performance enhancement of the scaffold over the flat structure. This discussion is saved for the complete system analysis at the end of this section. We further note the presence of similar spectral features in the absorption spectra of $\mathrm{Fe}_{2} \mathrm{O}_{3}$ when deposited on the inverse opal scaffold, which we often correlate to crystallinity. This, coupled with the conformality 

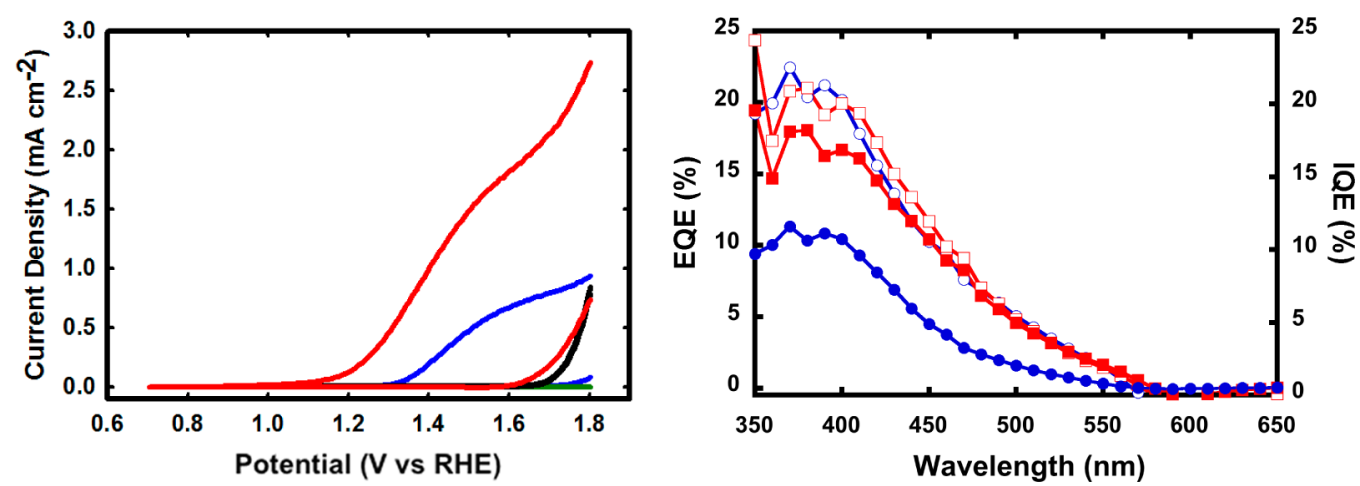

Figure 5. (a) Photocurrent density vs applied potential for FTO/aITO/hematite (blue), FTO/IO/aITO/hematite (red), FTO/IO/hematite (black) and FTO/IO/aITO (green). (b) EQE (closed circles) and IQE (open circles) plots of the same samples at $1.53 \mathrm{~V}$ vs RHE.

observed in SEM and EDS analysis, further suggests that the $\alpha$ $\mathrm{Fe}_{2} \mathrm{O}_{3}$ coated framework may be suitable for application as a comparatively efficient water oxidation photoanode.

The photoelectrochemical performance of a complete inverse opal photoanode is compared to an analogous flat FTO substrate (lacking inverse-opal scaffold) with identical ITO and $\mathrm{Fe}_{2} \mathrm{O}_{3}$ layers, Figure 5. A photocurrent density of $0.55 \mathrm{~mA} \mathrm{~cm}{ }^{-2}$ at $1.53 \mathrm{~V}$ vs RHE under simulated AM1.5 illumination is comparable to similar flat devices reported previously. ${ }^{9,13,43}$ Our champion nanolaminated scaffold-an inverse opal coated with $8 \mathrm{~nm}$ of ITO and $15 \mathrm{~nm}$ of $\alpha-\mathrm{Fe}_{2} \mathrm{O}_{3}$ - showed a photocurrent density of $1.60 \mathrm{~mA} \mathrm{~cm}^{-2}$ at $1.53 \mathrm{~V}$ vs RHE under the same photoelectrochemical conditions. This represents a 3fold enhancement over the flat electrode, and clearly demonstrates the value of the scaffold approach. At $1.23 \mathrm{~V}$ vs RHE (the thermodynamic water oxidation potential) the nanolaminate scaffold produced $0.2 \mathrm{~mA} \mathrm{~cm}{ }^{-2}$, a vast improvement over the control flat electrode. The exceptionally large enhancement (more than 100-fold) at this less anodic potential highlights a second benefit of the photoanode design-high surface area, which provides a larger specific surface area (per photocurrent density) through which photogenerated holes may oxidize water. This large improvement in onset potential has not been observed for related structures in the literature. The effect may derive from a hematite surface with exceptionally poor oxygen evolution kinetics under these process conditions that benefits more profoundly from a larger surface area. Alternatively, the improvement may reflect the particular effectiveness of this conductive scaffold in performing its intended role, i.e., to increase the useful light-harvesting efficiency and specific surface area of hematite without accelerating charge recombination. In the limit of equivalent recombination kinetics (and therefore similar quasi-Fermi level in the photoanode), a dramatically enhanced surface area is expected to result in a lower overpotential-a result we observe here experimentally.

The inverse opal scaffolds with $\alpha$ - $\mathrm{Fe}_{2} \mathrm{O}_{3}$ that lack a conformal ITO underlayer produce no photocurrent on this scale. The lack of significant photocurrent in these control samples is somewhat surprising but not wholly unexpected given the long, narrow, and tortuous path that electrons must travel through the micrometer-scale thickness of this electrode. A longer nucleation delay for ALD of $\mathrm{Fe}_{2} \mathrm{O}_{3}$ on the silica surface may also produce a film of $<10 \mathrm{~nm}$ thickness from which we observe negligible photocurrent, as previously discussed. The samples do, however, show a dark current onset around $1.6 \mathrm{~V}$ vs RHE that is similar to other high surface area electrodes. The ITO- coated inverse opals showed no photocurrent (as expected) but produced a significantly greater dark current density than flat ITO or FTO electrodes, albeit on a significantly reduced scale relative to the hematite-coated electrode (flat ITO/FTO electrode data not shown). This result again demonstrates the accelerated rate of the water oxidation reaction (per unit of projected surface area) obtainable on high specific-surface-area substrates.

The flat photoanode exhibits a maximum EQE of $11 \%$ near $400 \mathrm{~nm}$, Figure $5 \mathrm{~b}$, at $1.53 \mathrm{~V}$ vs RHE. The EQE for the nanolaminate scaffold, however, was much higher, yielding values approaching $18 \%$ at short wavelengths. Overall, a two- to 5-fold improvement in EQE compared to the flat electrode geometry is observed over the range from 350 to $525 \mathrm{~nm}$, consistent with the improvement in white light photocurrent performance. EQEs are the product of the absorptance (the simple ratio of the radiation absorbed by the active layer to that incident upon it, also called the light-harvesting efficiency) and the internal quantum efficiency (IQE). Thus the IQE values were calculated by dividing EQE values by the absorptance measured at each wavelength. IQE values for the flat films reach $20 \%$ near $400 \mathrm{~nm}$, and showing what has become the typical wavelength dependence for $\mathrm{Fe}_{2} \mathrm{O}_{3}$-dropping steadily as the wavelength approaches the absorption onset.

By analyzing the ratio of performance parameters between identical device stacks deposited on the high area framework vs flat platforms, the precise source of improvement-as well as deleterious effects of the scaffold-may be established. The most obvious benefit of depositing on the scaffold is significantly greater optical extinction, which is easily visible to the naked eye and clearly realized when examining the ratio of absorbance values attributable to $\mathrm{Fe}_{2} \mathrm{O}_{3}$, as shown in Figure 6. The enhancement of absorbance expected across the blue and green spectral regions is largely realized, with nearly a 10fold improvement between 500 and $550 \mathrm{~nm}$ (see Figure S3 in the Supporting Information). We note that the absorbance enhancement was determined for FTO/IO/aITO/15 nmFe $\mathrm{O}_{3}$ vs $\mathrm{FTO} / \mathrm{aITO} / 15 \mathrm{nmFe}_{2} \mathrm{O}_{3}$, for which the absorbance is plotted in Figure $\mathrm{S} 3 \mathrm{a}$ in the Supporting Information. A nominally similar enhancement is also observed for the FTO/IO/15 $\mathrm{nmFe}_{2} \mathrm{O}_{3}$ sample in the region between 350 and $450 \mathrm{~nm}$, indicating that competitive absorption from the aITO layer is minimal. The ratio of absorptance values follows a similar trend but is less pronounced because of the logarithmic relationship between absorption and transmission. Most strikingly, the enhancement in EQE almost exactly tracks the enhancement in spectral absorptance. This constitutes 


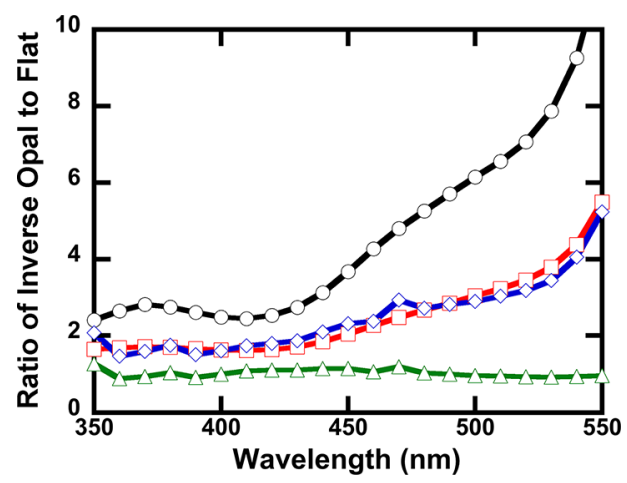

Figure 6. Ratio of the absorbance (black), absorptance (red), EQE (blue), and IQE (green) for inverse opal scaffold vs flat substrates coated with $8 \mathrm{~nm}$ ITO plus $15 \mathrm{~nm} \mathrm{Fe} \mathrm{O}_{3}$.

compelling evidence that the nanolaminate scaffold design approach described herein is effective: an increase in photocurrent density is realized and is directly proportional to the improvement in absorptance. Recall that this is clearly not the case for enhancements to absorptance produced via increasing the thicknesses of hematite films built on a unity roughness (flat) substrate, for which the EQE quickly saturates. The ratio of IQEs presented in Figure 6 is nearly uniform across the range of wavelengths absorbed, and is an equivalent way of demonstrating that the hematite coatings contributing to optical absorption from deep within the conductive scaffold are equally capable of photo-oxidizing water as identical hematite films grown directly on a planar electrode.

A full accounting of photons incident on flat and nanolaminate photoanodes, Figure 7, is instructive for identifying those improvements most needed to reach near-unity $\mathrm{EQE}$
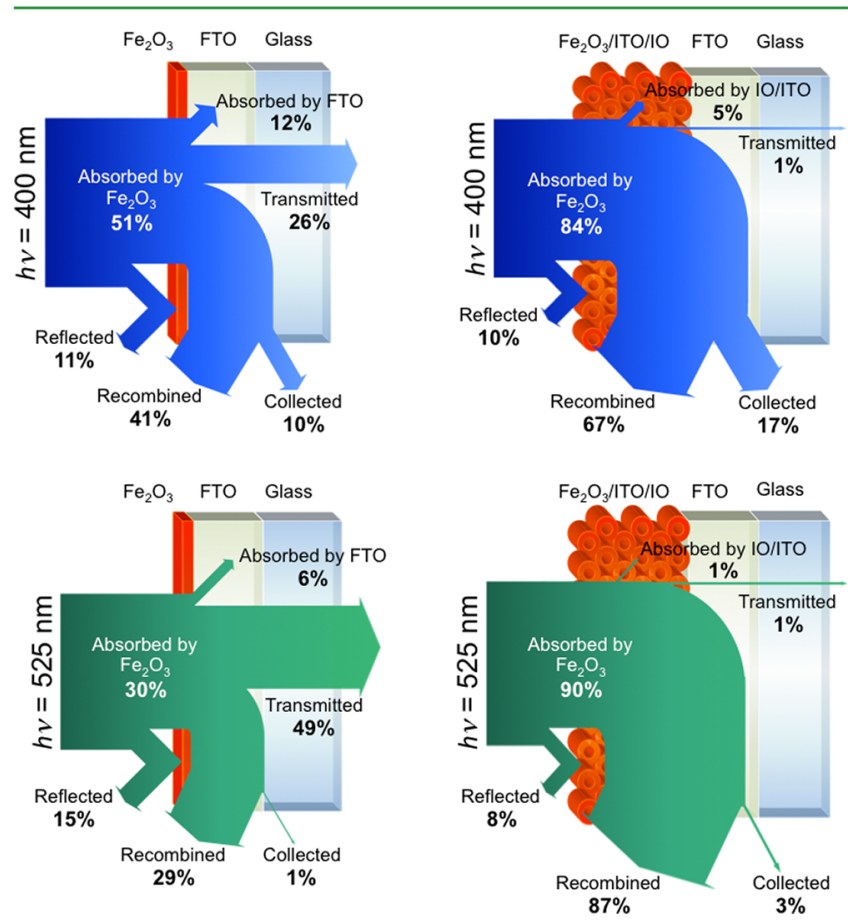

Figure 7. Illustration of average $400 \mathrm{~nm}$ (top) and $525 \mathrm{~nm}$ (bottom) photon absorption, transmission, and conversion for flat substrates (left) with $15 \mathrm{~nm} \mathrm{Fe}_{2} \mathrm{O}_{3}$ and inverse opal scaffolds (right) coated with $8 \mathrm{~nm}$ ITO plus $15 \mathrm{~nm} \mathrm{Fe}_{2} \mathrm{O}_{3}$. The photoanode schematics are for illustration purposes only and not drawn to scale. across the visible spectrum. For all samples, reflection losses are between 8 and 15\%. These are acceptable losses at this stage of development; they may be largely remedied with antireflective coatings in the future. For flat photoanodes with a compact hematite layer, much of the light passes through the sample because of insufficient absorption from such a thin film. The absorptance of flat hematite is only $51 \%$ at $400 \mathrm{~nm}$, and less than $30 \%$ at wavelengths greater than $525 \mathrm{~nm}$ despite a nominal bandgap of $2.1 \mathrm{eV}$-equivalent to photons with wavelength $590 \mathrm{~nm}$, Figure S4a in the Supporting Information. Our EQE experiments reveal that only $10 \%$ of $400 \mathrm{~nm}$ photon and $1 \%$ of $525 \mathrm{~nm}$ photons incident ultimately result in photocurrent. The remaining 41 and $29 \%$ of the total incident photons that have been absorbed, respectively, are lost to recombination.

For the nanolaminate-coated inverse opal scaffolds investigated here, almost no light was transmitted through the photoanodes (at $\lambda=525 \mathrm{~nm}$ or less). By modeling the competitive absorption between the active layer and the underlying ITO film on the inverse opal scaffold (by calculating attenuation for alternating layers of each with their respective absorption coefficients) we find that the active layer accounts for $94 \%$ of the absorbed light at $400 \mathrm{~nm}$ and $99 \%$ of absorbed light at $525 \mathrm{~nm}$. Therefore, $84 \%$ of $400 \mathrm{~nm}$ light incident on the sample is harvested by the active layer, as is $90 \%$ of incident 525 $\mathrm{nm}$ light. Overall, the fraction of incident light absorbed by the ITO was approximately $5 \%$ at $400 \mathrm{~nm}$ and only $1 \%$ at 525 $\mathrm{nm}^{44}$

This careful "photon accounting" reveals that while overcoming the disparate light and charge-collection distance requirements in hematite is challenging, a robust and flexible solution is now tenable. In contrast, the efficient conversion of absorbed photons to usefully extracted charge, even for those photons absorbed in ultrathin films over which a potential gradient is present to assist in charge collection, has significant room for improvement. For $400 \mathrm{~nm}$ photons, the highest IQEs reported to date are still less than $50 \%$, while at $550 \mathrm{~nm}$ IQEs are limited to less than $20 \%{ }^{13,18,45,46}$ The implications for this study are that while an absorptance enhancement of $>5$ is observed at wavelengths approaching $550 \mathrm{~nm}$ (see Figure 6), the characteristically poor IQE values at these wavelengths result in marginal contributions to the overall photocurrent enhancement. As a consequence, the white light photocurrent enhancement is limited to 3 -fold, despite the great potential for absorptance improvement at longer wavelengths (where the absorptivity is weakest). Clearly, modest IQEs severely limit the efficiency of hematite thin films for water oxidation applications, more so than any other loss. One potential path to IQE improvement would be the addition of an oxygenevolution catalysis to the hematite surface, whereby the photoelectrochemical reaction might more effectively compete with recombination. However, catalytic species with even modest optical densities in the visible region are not amenable to this design, as they too would compete for light needed for photocurrent generation. A second route to improve IQE includes tuning of the space-charge layer via intentional doping, so as to produce greater potential gradients across which photogenerated charges may be separated. These and related studies of well-defined hematite structures are under investigation in our laboratories. 


\section{CONCLUSIONS}

Porous and transparent inverse-opal frameworks coated with ultrathin ITO films by ALD are attractive candidates as conductive scaffolds (i.e., distributed current collectors) for photocatalytic semiconductor coatings. The electrical and optical properties of the scaffolds are surprisingly stable to both harsh annealing conditions and the strongly oxidizing conditions associated with water splitting. The highly transparent scaffolds were employed as high surface area frameworks on which to deposit $\mathrm{Fe}_{2} \mathrm{O}_{3}$ with thickness commensurate with the expected hole collection distance. Compared to an identical nanolaminate stack without scaffold, the onset of water oxidation is shifted by $-200 \mathrm{mV}$ and the photocurrent at $1.53 \mathrm{~V}$ vs RHE is tripled. Improvements in light-harvesting efficiency (relative to flat photoanodes) are correlated with nearly identical increases in EQE over the wavelength range from 350 to $550 \mathrm{~nm}$. The materials flexibility of this approach is an advantage over related, recently reported frameworks. Combined with rapid advances in ALD-derived materials quality, this approach should allow for additional improvements to hematite and similar photoanodes relevant to solar fuel production. A careful photon accounting of the optically dense photoanodes reveals that although nanolaminating TCOs and hematite over porous scaffolds results in significant improvements in photoelectrochemical efficiency for water oxidation, markedly better performance will be realized only once a route to improved IQEs has been identified.

\section{ASSOCIATED CONTENT}

\section{S Supporting Information}

Additional SEM/EDS line-scans, absorption spectra, and absorptance plots. This material is available free of charge via the Internet at http://pubs.acs.org.

\section{AUTHOR INFORMATION}

\section{Corresponding Author}

*E-mail: j-hupp@northwestern.edu (J.T.H.); martinson@anl. gov (A.B.F.M.).

\section{Author Contributions}

"These authors contributed equally.

\section{Notes}

The authors declare no competing financial interest.

\section{ACKNOWLEDGMENTS}

This work was supported as part of the ANSER Center, an Energy Frontier Research Center funded by the U.S. Department of Energy, Office of Science, Office of Basic Energy Sciences under Award DE-SC0001059. A portion of the research was performed at Argonne National Laboratory, a U.S. Department of Energy, Office of Science, Laboratory operated under Contract DE-AC02-06CH11357 by UChicago Argonne, LLC. A portion of the electron microscopy was accomplished at the Electron Microscopy Center for Materials Research at Argonne National Laboratory, a U.S. Department of Energy Office of Science Laboratory operated under Contract DEAC02-06CH11357 by UChicago Argonne, LLC. Addition electron microscopy was performed in the EPIC and KECKII facilities of the NUANCE Center at Northwestern University. The NUANCE center is supported by NSFNSEC, NSF-MRSEC, the KECK Foundation, the state of Illinois, and Northwestern University.

\section{ABBREVIATIONS}

ALD, atomic layer deposition; ITO, tin-doped indium oxide; OER, oxygen evolution reaction; FTO, fluorine-doped tin oxide; EQE, external quantum efficiency; IQE, internal quantum efficiency; LHE, light harvesting efficiency

\section{REFERENCES}

(1) Lewis, N. S.; Nocera, D. G. Proc. Natl. Acad. Sci. U.S.A. 2006, 103 (43), 15729-15735.

(2) Centi, G.; Perathoner, S. ChemSusChem 2010, 3 (2), 195-208.

(3) Roy, S. C.; Varghese, O. K.; Paulose, M.; Grimes, C. A. ACS Nano 2010, 4 (3), 1259-1278.

(4) Song, W. J.; Chen, Z. F.; Brennaman, M. K.; Concepcion, J. J.; Patrocinio, A. O. T.; Iha, N. Y. M.; Meyer, T. J. Pure Appl. Chem. 2011, 83 (4), 749-768.

(5) Walter, M. G.; Warren, E. L.; McKone, J. R.; Boettcher, S. W.; Mi, Q. X.; Santori, E. A.; Lewis, N. S. Chem. Rev. 2010, 110 (11), 64466473.

(6) Xing, J.; Fang, W. Q.; Zhao, H. J.; Yang, H. G. Chem. Asian J. 2012, 7 (4), 642-657.

(7) Hamann, T. W. Dalton Trans. 2012, 41 (26), 7830-7834.

(8) Katz, M. J.; Riha, S. C.; Jeong, N. C.; Martinson, A. B. F.; Farha, O. K.; Hupp, J. T. Coord. Chem. Rev. 2012, 256 (21-22), 2521-2529.

(9) Klahr, B.; Gimenez, S.; Fabregat-Santiago, F.; Bisquert, J.; Hamann, T. W. Energy Environ. Sci. 2012, 5 (6), 7626-7636.

(10) Klahr, B. M.; Martinson, A. B. F.; Hamann, T. W. Langmuir 2011, 27 (1), 461-468.

(11) Lin, Y.; Yuan, G.; Sheehan, S.; Zhou, S.; Wang, D. Energy Environ. Sci. 2011, 4 (12), 4862-4869.

(12) Lin, Y. J.; Xu, Y.; Mayer, M. T.; Simpson, Z. I.; McMahon, G.; Zhou, S.; Wang, D. W. J. Am. Chem. Soc. 2012, 134 (12), 5508-5511.

(13) Lin, Y. J.; Zhou, S.; Sheehan, S. W.; Wang, D. W. J. Am. Chem. Soc. 2011, 133 (8), 2398-2401.

(14) Peter, L. M.; Wijayantha, K. G. U.; Tahir, A. A. Faraday Discuss. 2012, 155, 309-322.

(15) Sivula, K.; Le Formal, F.; Grätzel, M. ChemSusChem 2011, 4 (4), $432-449$.

(16) Tartaj, P.; Morales, M. P.; Gonzalez-Carreño, T.; VeintemillasVerdaguer, S.; Serna, C. J. Adv. Mater. 2011, 23 (44), 5243-5249.

(17) Dotan, H.; Sivula, K.; Gratzel, M.; Rothschild, A.; Warren, S. C. Energy Environ. Sci. 2011, 4 (3), 958-964.

(18) Hisatomi, T.; Dotan, H.; Stefik, M.; Sivula, K.; Rothschild, A.; Grätzel, M.; Mathews, N. Adv. Mater. 2012, 24 (20), 2699-2702.

(19) Kennedy, J. H.; Frese, K. W. J. Electrochem. Soc. 1978, 125 (5), 709-714.

(20) Dare-Edwards, M. P.; Goodenough, J. B.; Hamnett, A.; Trevellick, P. R. J. Chem. Soc., Faraday Trans. 1 F 1983, 79 (9), 2027-2041.

(21) Frese, K. W.; Kennedy, J. H. J. Electrochem. Soc. 1978, 125 (3), C160-C160.

(22) Kennedy, J. H.; Frese, K. W. J. Electrochem. Soc. 1977, 124 (3), C130-C130.

(23) Bjorksten, U.; Moser, J.; Gratzel, M. Chem. Mater. 1994, 6 (6), $858-863$.

(24) Beermann, N.; Vayssieres, L.; Lindquist, S. E.; Hagfeldt, A. J. Electrochem. Soc. 2000, 147 (7), 2456-2461.

(25) Chemelewski, W. D.; Hahn, N. T.; Mullins, C. B. J. Phys. Chem. C 2012, 116 (8), 5256-5262.

(26) Hahn, N. T.; Mullins, C. B. Chem. Mater. 2010, 22 (23), 64746482.

(27) Hahn, N. T.; Ye, H.; Flaherty, D. W.; Bard, A. J.; Mullins, C. B. ACS Nano 2010, 4 (4), 1977-1986.

(28) Kay, A.; Cesar, I.; Gratzel, M. J. Am. Chem. Soc. 2006, 128 (49), 15714-15721.

(29) Martinson, A. B. F.; Elam, J. W.; Liu, J.; Pellin, M. J.; Marks, T. J.; Hupp, J. T. Nano Lett 2008, 8 (9), 2862-2866.

(30) Williams, V. O.; Jeong, N. C.; Prasittichai, C.; Farha, O. K.; Pellin, M. J.; Hupp, J. T. ACS Nano 2012, 6 (7), 6185-6196. 
(31) Yang, Z.; Gao, S.; Li, T.; Liu, F.-Q.; Ren, Y.; Xu, T. ACS Appl. Mater. Interface 2012, 4 (8), 4419-4427.

(32) Stefik, M.; Cornuz, M.; Mathews, N.; Hisatomi, T.; Mhaisalkar, S.; Grätzel, M. Nano Lett. 2012, 12 (10), 5431-5435.

(33) Gemmer, J.; Hinrichsen, Y.; Abel, A.; Bachmann, J. J. Catal. 2012, 290 (0), 220-224.

(34) Hatton, B.; Mishchenko, L.; Davis, S.; Sandhage, K. H.; Aizenberg, J. Proc. Natl. Acad. Sci. U.S.A. 2010, 107 (23), 1035410359.

(35) Williams, V. O.; Demarco, E.; Libera, J. A.; Riha, S. C.; Farha, O. K.; Martinson, A. B. F.; Elam, J. W.; Pellin, M. J.; Hupp, J. T. Manuscript in preparation 2012.

(36) Martinson, A. B. F.; DeVries, M. J.; Libera, J. A.; Christensen, S. T.; Hupp, J. T.; Pellin, M. J.; Elam, J. W. J. Phys. Chem. C 2011, 115 (10), 4333-4339.

(37) Escrig, J.; Bachmann, J.; Jing, J.; Daub, M.; Altbir, D.; Nielsch, K. Phys. Rev. B 2008, 77 (21), 214421.

(38) Chong, Y. T.; Gorlitz, D.; Martens, S.; Yau, M. Y. E.; Allende, S.; Bachmann, J.; Nielsch, K. Adv. Mater. 2010, 22 (22), 2435-2439.

(39) Chong, Y. T.; Yau, E. M. Y.; Nielsch, K.; Bachmann, J. Chem. Mater. 2010, 22 (24), 6506-6508.

(40) Souza, F. L.; Lopes, K. P.; Longo, E.; Leite, E. R. Phys. Chem. Chem. Phys. 2009, 11 (8), 1215-1219.

(41) King, P. D. C.; Veal, T. D. J. Phys.: Condens. Matter 2011, 23, 334214.

(42) Elam, J. W.; Baker, D. A.; Martinson, A. B. F.; Pellin, M. J.; Hupp, J. T. J. Phys. Chem. C 2008, 112 (6), 1938-1945.

(43) Klahr, B.; Gimenez, S.; Fabregat-Santiago, F.; Hamann, T.; Bisquert, J. J. Am. Chem. Soc. 2012, 134 (9), 4294-4302.

(44) Absorption falloff was calculated as $10^{-\alpha d}$ assuming alternating layers of $30 \mathrm{~nm}$ of $\mathrm{Fe}_{2} \mathrm{O}_{3}$ and $20 \mathrm{~nm}$ ITO. Absorption coefficients used were calculated from absorption spectra in SI-2.

(45) Le Formal, F.; Grätzel, M.; Sivula, K. Adv. Funct. Mater. 2010, 20 (7), 1099-1107.

(46) Tilley, S. D.; Cornuz, M.; Sivula, K.; Grätzel, M. Angew. Chem., Int. Ed. 2010, 49 (36), 6405-6408. 